\title{
Podocyte Structural Parameters Do Not Predict Progression to Diabetic Nephropathy in Normoalbuminuric Type 1 Diabetic Patients
}

\author{
Tasma Harindhanavudhi ${ }^{\mathrm{a}} \quad$ Alicia Parks ${ }^{\mathrm{a}} \quad$ Michael Mauer ${ }^{\mathrm{a}, \mathrm{b}}$ \\ M. Luiza Caramori ${ }^{a, b}$ \\ Departments of ${ }^{\mathrm{a}}$ Medicine, and ${ }^{\mathrm{b}}$ Pediatrics, University of Minnesota, Minneapolis, Minn., USA
}

\author{
Key Words \\ Nephropathy $\cdot$ Normoalbuminuria $\cdot$ Podocyte $\cdot$ Type 1 \\ diabetes
}

\begin{abstract}
Background: Podocyte injury has been implicated in diabetic nephropathy (DN) ranging from normoalbuminuria to proteinuria in both type 1 and type 2 diabetes. Methods: To determine whether podocyte structural parameters predict DN risk in initially normoalbuminuric long-standing type 1 diabetic patients, we performed a nested case-control study in sex and diabetes duration-matched progressors (progression to proteinuria or ESRD, $n=10$ ), non-progressors (normoalbuminuric at follow-up, $n=10$ ), and non-diabetic controls $(\mathrm{n}=10)$. Results: $\mathrm{HbA}_{1 \mathrm{c}}$ and diastolic blood pressure were higher in progressors versus non-progressors. Podocyte number per glomerulus, numerical density of podocyte per glomerulus, and foot process width were not different among groups. The glomerular basement membrane width was greater in progressors versus non-progressors or controls, and in non-progressors versus controls. As expected, the mesangial fractional volume was greater in progressors and non-progressors versus controls, with no differences between progressors and non-progressors. Conclusion: This study does not indicate that podocyte structural changes are preconditions for later DN progression in initially normoal-
\end{abstract}

buminuric type 1 diabetic patients. However, this does not preclude an important role for podocyte injury at a later stage of DN.

(c) 2015 S. Karger AG, Basel

\section{Introduction}

Despite the recommendations for improved glycemic and blood pressure control and the widespread use of renin-angiotensin system blocking agents, the annual incidence of end-stage renal disease (ESRD) in patients with diabetes in the United States is steadily rising (http:// www.usrds.org/atlas.aspx, assessed on September 28, 2014) and diabetic nephropathy (DN) is currently the single most common cause of ESRD in the western world. Although microalbuminuria remains an important clinical indicator of increased DN risk, only $30-45 \%$ of patients with microalbuminuria progress to proteinuria over 5-10 years, and many spontaneously revert to normoalbuminuric or remain microalbuminuric on longterm follow-up [1]. Moreover, about 5\% of normoalbuminuric patients with at least 7 years of type 1 diabetes (T1D) will progress to proteinuria over the subsequent

T.H. and A.P. equally contributed to this paper.

\section{KARGER 125\%}

(c) 2015 S. Karger AG, Base

0250-8095/15/0415-0277\$39.50/0

E-Mail karger@karger.com

www.karger.com/ajn
M. Luiza Caramori, $\mathrm{MD}, \mathrm{PhD}$

420 Delaware Street S.E., MMC 101

Minneapolis, MN 55455 (USA)

E-Mail caram001@umn.edu 
6-10 years, whereas $17 \%$ will progress to microalbuminuria. These data suggest that urinary albumin, although a strong biomarker, is not a precise predictor of DN risk [1]. In fact, advanced glomerular structural lesions, which may overlap with the structural changes found in microalbuminuric and proteinuric patients, can be present in long-standing normoalbuminuric T1D patients [2,3], albeit that some of these normoalbuminuric patients may have reduced glomerular filtration rate (GFR) and/or hypertension $[4,5]$.

Injury to podocytes has been implicated in $\mathrm{DN}$ pathogenesis and progression. Increased foot process (FP) width has been observed in young normoalbuminuric T1D adolescents [6] and the widening of FP also significantly progressed in follow-up biopsies over 5 years [7]. T1D patients with microalbuminuria also have wider FP as compared with normoalbuminuric patients [8]. Moreover, the inverse of FP width, filtration slit length density, correlated very strongly $(\mathrm{r}=-0.80, \mathrm{p}<0.001)$ with albumin excretion rate (AER) in T1D subjects with a wide AER range [9]. We previously reported decreased podocyte attachment in proteinuric T1D patients [10]. Reduced podocyte number and/or podocyte density per glomerulus were reported in proteinuric type 2 diabetic (T2D) patients [11-15] and were found to be predictive of nephropathy progression $[14,15]$, whereas data on podocyte number per glomerulus in T1D individuals remained controversial [16, 17].

Few longitudinal studies have evaluated initially normoalbuminuric patients to determine the early renal structural changes associated with $\mathrm{DN}$ progression. We recently demonstrated that glomerular basement membrane (GBM) width was greater in normoalbuminuric T1D patients who progressed to proteinuria and ESRD as compared to those remaining normoalbuminuric after many years of follow-up [18]. This study aimed at determining whether podocyte structural parameters could predict progression to proteinuria and/or ESRD in these initially long-standing normoalbuminuric T1D patients.

\section{Subjects and Methods}

\section{Patients}

Kidney biopsies and renal functional studies were performed in 94 normoalbuminuric T1D research volunteers between 1982 and 2005 at the University of Minnesota. All had at least 5 years of T1D at time of biopsy, were 18 years or older, and were not on renin-angiotensin system blocking agents at the time the initial studies were conducted. The clinical and demographic characteristics of this cohort have been detailed elsewhere [18].

\section{Clinical Studies}

Clinical investigations were carried out in the General Clinical Research Center (GCRC) at the University of Minnesota by experienced research nurses. Blood pressure (BP) was the average of multiple ( $>15)$ standardized Dinamap BP measurements. Hypertension was defined as BP $>130 / 85 \mathrm{~mm} \mathrm{Hg}$ and/or use of anti-hypertensive drugs. AER was measured by fluorescent immunoassay [19] in at least two GCRC obtained 24-hour urine collections at baseline and at in least two timed overnight urine collections at follow-up. Normoalbuminuria was defined as AER $<20 \mu \mathrm{g} / \mathrm{min}$, microalbuminuria as $20-200 \mu \mathrm{g} / \mathrm{min}$ and proteinuria as $>200 \mu \mathrm{g} /$ min in at least 2 of 3 consecutive urine samples. Creatinine was measured by Jaffe's reaction. GFR was measured by iohexol plasma disappearance [20], iothalamate clearance [21] or estimated by the average of three carefully GCRC collected creatinine clearances $(\mathrm{CrCl})$. The mean of these multiple GCRC' $\mathrm{CrCl}$ has previously been shown to be strongly correlated with insulin clearance $(\mathrm{r}=0.92, \mathrm{p}<0.001)$ performed during the same GCRC admission [22]. Glycated hemoglobin was measured by a non-automated ion exchange chromatography micro-column from 1982 to 1986 $\left(\mathrm{HbA}_{1}\right.$ reference range: 5.5-8.5\%), by an automated high-performance ion-exchange liquid-chromatographic (HPLC; Biorad) from 1986-1992 $\left(\mathrm{HbA}_{1}\right.$ reference range: 5.1-7.3\%; $\mathrm{HbA}_{1 \mathrm{c}}$ reference range: $4.3-6.1 \%)$, and by the same method standardized to the Diabetes Control and Complications Trial (DCCT) values since then $\left(\mathrm{HbA}_{1}\right.$ reference range: 5.4-7.4\%; $\mathrm{HbA}_{1 \mathrm{c}}$ reference range: $4.3-6.0 \%)$. Results prior to 1992 were converted to current $\mathrm{HbA}_{1 \mathrm{c}}$ standards using published equations [23].

Follow-Up Studies. The protocol for the follow-up studies has been previously published [18]. Briefly, patients who were alive and could be contacted were asked to complete a questionnaire accessing their current clinical status. Patients who were on dialysis or had received a kidney transplant, as well as patients with proteinuria (urinary albumin $>200 \mu \mathrm{g} / \mathrm{min}$ ) on at least 2 out of 3 overnight urine samples, were classified as progressors. Patients who were on angiotensin converting enzyme inhibitors (ACEi) or angiotensin receptor blockers (ARB) at follow-up and were normoalbuminuric or microalbuminuric underwent a 2-month ACEi/ ARB drug washout and then had AER re-measured using the same protocol. Patients who remained normoalbuminuric after ACEi/ ARB washout (on those receiving these agents) were classified as non-progressors, as well as any patient not on these agents who remained normoalbuminuric at follow-up. During the washout period, BP was controlled as per protocol [18]. AER values at the end of the washout period were used to classify these patients as progressors or non-progressors.

Patients in whom follow-up information was not available were searched for in the United States Renal Data System and in the National Death Index databases. Relatives of deceased patients were also contacted.

As we previously reported [18], among the 82 patients in whom we were able to obtain follow-up information, 12 patients had developed proteinuria and/or progressed to ESRD (progressors; P) and 59 patients remained normoalbuminuric (non-progressors; NP). We thus designed a nested case-control study. Podocyte structural parameters were obtained in all $\mathrm{P}$, except for 2 subjects whose tissue was, on masked screening, considered inappropriate for these podocyte studies; this was based on the severity of tissue trauma-related artifacts, and in $10 \mathrm{NP}$, matched for sex, T1D duration and length of follow-up to the $\mathrm{P}$, as well as in 10 sex- and age- 
matched non-diabetic living kidney donor controls (C). The nested case-control design allows for the study of a smaller number of subjects without significant loss of statistical power as compared to studying the whole cohort. The living kidney donors agreed to research kidney biopsies obtained during transplant surgery, while their kidneys were still in situ. This study was approved by the Institutional Review Board on human research at the University of Minnesota and informed consent was obtained from all living participants.

\section{Renal Morphometric Measurements}

Kidney biopsies were processed for light and electron microscopy (EM) as previously detailed $[2,18]$. Semi-thin $(1 \mu \mathrm{m})$ sections of Epon embedded tissues were used to select the centermost glomerulus with intact Bowman's capsule in each block. Between 2 and 3 intact glomeruli from each subject were examined by a JEOL 100CX electron microscope (Tokyo, Japan) using a systematic uniform random sampling protocol. Overlapping digital electron microscopic images were used to construct a montage of glomerular profile at 3,600× magnification. Two masked observers evaluated these montages. Glomeruli with discernible mechanical artifacts such as marked glomerular tuft compression, extensive extrusion of proximal tubular cellular material into glomerular urinary or capillary luminal spaces, or rupture of GBM or Bowman's capsule were excluded. A single masked observer obtained all the stereological measurements.

The methods used for measuring GBM width, the fractional volume of glomeruli occupied by mesangium $(\mathrm{Vv}(\mathrm{Mes} / \mathrm{glom}))$ and the surface density of the peripheral GBM per glomerulus (Sv(PGBM/glom)) were described in detail elsewhere [2, 18]. Briefly, micrographs for measurement of GBM width and for mesangium composition were obtained by using a systematic uniform random sampling protocol at $11,000 \times$. Micrographs at $3,900 \times$ were constructed into a montage of the entire glomerular profile for measurements of $\mathrm{Vv}(\mathrm{Mes} / \mathrm{glom})$ and $\mathrm{Sv}(\mathrm{PGBM} / \mathrm{glom})$. GBM width was estimated by the orthogonal intercept method [24], Vv(Mes/glom) by point counting [25], and Sv(PGBM/glom) by the intercept method $[2,3]$.

Images at 29,000× were used for the measurement of podocyte structures. A line to demarcate the peripheral versus mesangial GBM was drawn where the parallelism between the capillary endothelial cells and GBM was lost. An unbiased counting frame $(224 \times 224 \mathrm{~mm})$ with fine parallel lines $4 \mathrm{~mm}$ apart with 1 coarse line per 7 fine lines was superimposed to the images as previously described [10]. Only peripheral podocyte-GBM interfaces where FP continuously covered GBM were used to calculate the podocyte FP width. The number of intercepts between the vertical lines and FP were counted on peripheral GBM (PGBM). The fractional surface of the number of intercepts was estimated as

$$
\mathrm{Ss}(\mathrm{X} / \mathrm{Z})=\Sigma \mathrm{I}_{\text {intact FP }} / \Sigma \mathrm{Iz}
$$

where $\mathrm{X}$ represents areas with intact $\mathrm{FP}, \mathrm{Z}$ is the reference area (PGBM), $\Sigma \mathrm{I}_{\text {intact }}$ FP is sum of the number of intercepts on intact FP, and $\Sigma I z$ is the number of intercepts on PGBM. The slit diaphragm length density per PGBM (Ls(Slit/PGBM)) was measured in areas with intact FP. This parameter was calculated as

$$
\mathrm{Ls}(\mathrm{Slit} / \mathrm{PGBM})=\Sigma \mathrm{Q}_{\text {Slit }} /\left(\sum \mathrm{I}_{\text {intact FP }} * \mathrm{~d}\right)
$$

Podocyte Injury in Type 1 Diabetes where $\mathrm{Q}_{\text {Slit }}$ is the number of filtration slit profiles in the counting frame, $I_{\text {intact }}$ FP is the number of the line intercepts with the area of intact FP, and d is the distance between the coarse lines. The reciprocal of this parameter is the average FP width.

Mean glomerular volume was estimated using the WeibelGomez method in light microscopy slides [13]. Tissue fixed in Zenker's solution and embedded in paraffin was serially cut into $5 \mu \mathrm{m}$ sections and stained with periodic acid Schiff s (PAS). A minimum of 15 glomeruli were measured. Digital EM images at 3,600× were used to measure podocyte nuclear density per glomerulus $(\mathrm{Nv}$ (Podo/glom $)$ ) by the Weibel-Gomez method as previously described [13]. The number of podocyte per glomerulus was determined by multiplying the mean glomerular volume by the podocyte density per glomerulus.

\section{Statistical Analysis}

Statistical analyses were conducted using SPSS version 18.0. Data were expressed as mean $\pm \mathrm{SD}$, median and range, or number of subjects with a given characteristic. AER values, not normally distributed, were logarithmically transformed before analyses and expressed as median and range. Comparisons among groups were performed by the analysis of variance (ANOVA) method followed by the least significant difference (LSD) for multiple comparisons as indicated. $\mathrm{p}$ values $<0.05$ were considered statistically significant.

\section{Results}

Subjects at baseline were $28.7 \pm 7.3$ years old and had T1D for $15.2 \pm 6.9$ years. By design, there were no differences in sex distribution among groups, or differences in diabetes duration and length of follow-up between the diabetic groups. Baseline AER, GFR and frequency of hypertension were not different between progressors $(\mathrm{P})$ and non-progressors (NP), while $\mathrm{P}$ had significantly higher $\mathrm{HbA}_{1 \mathrm{c}}(\mathrm{p}=0.001)$ and diastolic blood pressure $(\mathrm{p}=0.04)$ at baseline than NP (table 1$)$.

Reflecting the findings of the larger cohort, $\mathrm{P}$ had greater GBM width than NP $(p=0.036)$ and non-diabetic controls $(C)(p<0.001)$, while NP also had greater GBM width than $\mathrm{C}(\mathrm{p}=0.033$; table 2$)$. Mesangial fractional volume per glomerulus $(\mathrm{Vv}(\mathrm{Mes} / \mathrm{glom}))$ was greater in $\mathrm{P}$ and NP versus $\mathrm{C}$ subjects $(\mathrm{p}=0.003$ and $\mathrm{p}<0.001$, respectively), but not statistically significantly different between $\mathrm{P}$ and NP. The peripheral glomerular capillary filtration surface density (Sv(PGBM/glom)) was not different among groups (table 2).

The mean glomerular volume, podocytes FP width, numerical density of podocytes per glomerulus ( $\mathrm{Nv}$ (Podo/ glom)) and number of podocytes per glomerulus were not statistically significantly different among groups (table 2). 
Table 1. Baseline demographic and clinical characteristics of normoalbuminuric type 1 diabetic subjects classified as progressors or nonprogressors and non-diabetic controls

\begin{tabular}{|c|c|c|c|c|}
\hline & $\begin{array}{l}\text { Progressors } \\
(\mathrm{n}=10)\end{array}$ & $\begin{array}{l}\text { Non-progressors } \\
(\mathrm{n}=10)\end{array}$ & $\begin{array}{l}\text { Controls } \\
(\mathrm{n}=10)\end{array}$ & $\mathrm{p}$ value \\
\hline Age, years & $26.3 \pm 7.9$ & $31.0 \pm 6.3$ & $26.5 \pm 8.6$ & 0.317 \\
\hline Age at diagnosis, years & $11.5 \pm 6.9$ & $15.4 \pm 8.5$ & - & 0.274 \\
\hline Diabetes duration, years & $14.8 \pm 7.7$ & $15.6 \pm 6.4$ & - & 0.803 \\
\hline Follow-up, years & $15.0 \pm 7.3$ & $17.3 \pm 5.2$ & - & 0.808 \\
\hline $\mathrm{SBP}, \mathrm{mm} \mathrm{Hg}$ & $113.7 \pm 9.8$ & $106.5 \pm 9.3$ & - & 0.11 \\
\hline $\mathrm{DBP}, \mathrm{mm} \mathrm{Hg}$ & $74.1 \pm 7.9$ & $70.0 \pm 6.7$ & - & 0.04 \\
\hline $\mathrm{AER}, \mu \mathrm{g} / \mathrm{min}$ & $5.50(0.01-15.79)$ & $4.24(0.01-10.76)$ & - & 0.803 \\
\hline $\mathrm{GFR}, \mathrm{ml} / \mathrm{min} / 1.73 \mathrm{~m}^{2}$ & $106.7 \pm 26.5$ & $101.0 \pm 23.7$ & - & 0.618 \\
\hline
\end{tabular}

Data are mean $\pm \mathrm{SD}$, median and range, or number of subjects with a given characteristic. $\mathrm{Hb}_{1 \mathrm{c}}=\mathrm{Glycated}$ hemoglobin $\mathrm{A}_{1 \mathrm{c}} ; \mathrm{SBP}=$ systolic blood pressure; $\mathrm{DBP}=$ diastolic blood pressure; $\mathrm{AER}=$ albumin excretion rate; $\mathrm{GFR}=$ glomerular filtration rate; $\mathrm{NA}=$ not applicable, similar by design.

Table 2. Baseline glomerular and podocyte structural parameters in normoalbuminuric type 1 diabetic subjects classified as progressors, non-progressors and non-diabetic controls

\begin{tabular}{|c|c|c|c|c|}
\hline & $\begin{array}{l}\text { Progressors } \\
(\mathrm{n}=10)\end{array}$ & $\begin{array}{l}\text { Non-progressors } \\
(\mathrm{n}=10)\end{array}$ & $\begin{array}{l}\text { Controls } \\
(\mathrm{n}=10)\end{array}$ & $\begin{array}{l}\mathrm{p} \text { value } \\
\text { ANOVA }\end{array}$ \\
\hline GBM width, nm & $522.8 \pm 89.2^{\mathrm{a}, \mathrm{b}}$ & $448.1 \pm 85.2^{\mathrm{c}}$ & $371.7 \pm 45.3$ & 0.001 \\
\hline Sv(PGBM/glom $)$ & $0.12 \pm 0.02$ & $0.12 \pm 0.02$ & $0.12 \pm 0.02$ & 0.792 \\
\hline Filtration surface per glomerulus, $\times 10^{-3} \mathrm{~m}^{2}$ & $230.5 \pm 86.9$ & $186.6 \pm 69.2$ & $231.1 \pm 148.1^{*}$ & 0.560 \\
\hline $\mathrm{GV}, \times 10^{6} \mu \mathrm{m}^{3}$ & $1.83 \pm 0.54$ & $1.58 \pm 0.48$ & $1.79 \pm 0.1^{*}$ & 0.689 \\
\hline $\mathrm{Nv}($ Podo/glom $), \mathrm{n} / \times 10^{6} \mu \mathrm{m}^{3}$ & $208.0 \pm 43.4$ & $243.0 \pm 87.7$ & $268.0 \pm 101.7$ & 0.272 \\
\hline Podo N/glom & $378.5(213.8-457.1)$ & $386.5(190.5-691.8)$ & $318.5(234.4-1,778.2)^{*}$ & 0.770 \\
\hline
\end{tabular}

Data are means $\pm \mathrm{SD}$, median and range. $\mathrm{GBM}=$ Glomerular basement membrane; $\mathrm{Vv}(\mathrm{Mes} /$ glom $)=$ mesangial fractional volume per glomerulus; $\mathrm{Sv}(\mathrm{PGBM}$ /glom $)$ = glomerular filtration surface density per glomerulus; $\mathrm{FP}=$ foot process; $\mathrm{GV}=$ glomerular volume; $\mathrm{Nv}($ Podo/glom $)=$ podocyte density per glomerulus; Podo N/glom = podocyte number per glomerulus. ${ }^{\mathrm{a}} \mathrm{p}=0.036$ progressors versus non-progressors. ${ }^{\mathrm{b}} \mathrm{p}<0.001$ progressors versus controls. ${ }^{\mathrm{c}} \mathrm{p}=0.033$ non-progressors versus controls. ${ }^{\mathrm{d}} \mathrm{p}=0.002$ progressors versus controls. ${ }^{\mathrm{e}} \mathrm{p}<0.001$ non-progressors versus controls. ${ }^{*} \mathrm{n}=8$.

\section{Discussion}

$\mathrm{DN}$, in some patients, is a progressive but initially clinically silent disease, thus serious renal structural abnormalities often develop and may advance while a patient remains normoalbuminuric $[2,3]$. Extracellular matrix accumulation, represented by mesangial expansion and an increase in GBM and tubular basement membrane (TBM) width, is central to the pathogenesis of DN and strongly correlated with renal functional abnormalities
$[2,26,27]$. Podocyte injury has also been recognized as a potential contributor to DN. In this study, we aimed at determining whether baseline podocyte structural parameters were independent predictors of progression to proteinuria and/or ESRD in initially normoalbuminuric T1D patients.

We found that podocyte structural parameters were not independent predictors of progression to proteinuria and/or ESRD in these normoalbuminuric T1D patients. Consistent with our recent publication in the total co- 
hort, we found greater GBM width and higher $\mathrm{HbA}_{1 c}$ at baseline in normoalbuminuric T1D patients who later progressed to clinically significant DN [18]. Although glomerular structure is normal at the onset of diabetes, as many as half of long-standing T1D patients who remain normoalbuminuric can have substantial glomerular lesions, including increased GBM and TBM width, mesangial expansion and reduced glomerular filtration surface density $[1,22,27,28]$. It is important to note that the lesions seen in normoalbuminuric patients may overlap in severity with those seen in microalbuminuric and proteinuric patients $[2,3,29]$. Moreover, Drummond et al. demonstrated that GBM width expanded linearly with increasing duration of T1D, particularly during the first 5-10 years of disease, whereas $\mathrm{Vv}(\mathrm{Mes} / \mathrm{glom})$ appeared to increase slowly, if at all, during the initial 15 years [30], rising thereafter. This suggests that the thickening of GBM may be the only readily detectable structural abnormality seen in the first 10 years of $\mathrm{DN}$. $\mathrm{Vv}$ (Mes/glom) and GBM width are highly correlated with AER $[26,31]$. GBM width was a predictor of development of microalbuminuria over the next 7 years in normoalbuminuric children and adolescents with about 10 years of T1D duration [32]. Thus, it is not unexpected that increased GBM width also predicts progression to proteinuria and/ or ESRD in initially normoalbuminuric long-standing T1D patients.

It is thought that podocytes do not readily replicate. Widening of FP width has been observed in proteinuric and microalbuminuric T1D patients and in proteinuric T2D patients [8-11]. FP width also correlates directly with AER $[8-10,27]$. Although the widening of FP width has previously been identified in young normoalbuminuric T1D patients when compared to living kidney donor controls [6], and FP width was shown to subsequently increase in these patients in 5-year follow-up biopsies [7], the widening of FP has not been observed in other cohorts evaluating normoalbuminuric middle aged subjects with T1D [9] or T2D [11] as compared to non-diabetic controls. In this study, we found no group differences between progressors and non-progressors in FP width at baseline, suggesting that the widening of FP may be subtle and short-lived, or nonexistent, at these earlier stages of DN.

When progressive podocyte damage occurs, not only are FP widened, but podocyte loss may also occur. A reduction in podocyte number has been described in both T1D and T2D. Thus, microalbuminuric and proteinuric T2D patients were reported to have statistically significant reductions in podocyte number per glomerulus and podocyte density per glomerulus that were inversely correlated with increased AER [12]. Similarly, reduced podocyte number and numerical density were observed in microalbuminuric and proteinuric Northern Italian T2D patients, and podocyte numerical density also inversely correlated with AER [13] in this cross-sectional study. Among microalbuminuric Pima Indian T2D patients, the number of podocytes per glomerulus was the strongest correlate of changes in urinary albumin excretion over 4 years [14], while the correlation between $\mathrm{Vv}$ (Mes/glom) and changes in urinary albumin levels was almost as strong. However, multiple regression analyses to examine whether the number of podocytes per glomerulus and $\mathrm{Vv}$ (Mes/glom) were independent predictors of changes in urinary albumin were not reported [14]. Both podocyte numerical density and the number of podocytes per glomerulus were found to be significantly lower in proteinuric as compared to normoalbuminuric T2D patients [11]. However, podocyte number per glomerulus was more variable in T1D studies. The podocyte number per glomerulus correlated inversely with AER in proteinuric but not in microalbuminuric normotensive T1D patients [17], whereas podocyte numerical density but not podocyte number per glomerulus was reduced in proteinuric T1D patients as compared to non-diabetic control subjects [17]. Our findings also contradict those of Steffes et al., the only study that reports a statistically significant reduction in the number of podocytes in normoalbuminuric T1D patients [16]. The difference may be explained by use of different methodology for determining glomerular volume. Our study used the Weibel-Gomez technique that takes shape into consideration, whereas the method used in Steffes et al. was Cavalieri, which is considered a gold standard because it assesses glomerular volume without assumptions on shape, but requires knowledge of section thickness and complete sectioning of the glomerulus. Thus, it is labor intensive and has the potential to exhaust irreplaceable research materials.

The podocyte number per glomerulus in this study, either in patients with diabetes or in controls, was lower than those of other studies [17], with no differences among groups. However, podocyte numerical density in controls was, in average, greater in this study than in other studies [17]. Several factors contribute to podocyte number and density. Podocyte number per glomerulus is obtained by multiplying the podocyte density per glomerulus by the glomerular volume, and glomerular volumes were lower in the present study as compared to some of these previous reports $[7,17]$. Differences in the 
methods used for tissue preparation can clearly explain the differences between our results and others. In our study, tissues were paraffin embedded, and thus prone to glomerular shrinkage, leading to relatively smaller glomerular volumes [33]. This method was in contrast to that of Perrin et al. [7], who used plastic-embedded tissues, which are associated with a low degree of shrinkage. Since these two methods were highly correlated [13], we applied a correction factor (2.09) to our paraffin embedded glomerular volume data in order to convert these to plastic embedded values, and we found, indeed, that our glomerular volumes were comparable to published values, obtained from plastic embedded tissues $[7,11,13,16$, 17]. As expected, since the correction factor was applied to the glomerular volume measurements of all participants, the results of our main analyses did not change. In fact, we have not found increased glomerular volume in our studies of large numbers of normoalbuminuric T1D patients, while this is commonly found in normoalbuminuric T2D patients [11].

In summary, our studies found that podocyte structural parameters did not predict progression to proteinuria and/or ESRD in long-standing normoalbuminuric T1D patients. These results do not rule out a possible role for podocyte structural changes as progression promoters at later DN stages. It is possible that more precise and accurate estimates of podocyte numerical density and/or glomerular volume could detect subtle group differences, which were not seen here. It is also possible that other podocyte structural parameters, for example, podocyte mitochondrial size and/or number, or histochemical studies of slit pore or adhesion molecules, might have provided early podocyte predictors of $\mathrm{DN}$ risk in these long-standing normoalbuminuric patients. However, from our studies, only glycemic control and
GBM width emerged as significant independent predictors of progression to $\mathrm{DN}$ in normoalbuminuric patients with long-standing T1D. Although the design of the current study (nested case-control study) allows for the study of a smaller number of controls without loss of statistical power, it is conceivable that the study of cohorts where a larger number of subjects reached meaningful endpoints could yield greater power to detect group differences. Although these would be important scientific contributions, they are unlikely to happen since, to the best of our knowledge, this is the largest cohort study where kidney biopsies were performed in normoalbuminuric T1D research volunteers with a follow-up long enough as to allow for clinical DN and ESRD to develop.

\section{Acknowledgments}

We thank Mr. Thomas Groppoli, Ms. Ann Palmer and Ms. Frida Maiers for technical support for renal structural studies; Ms. Ashley Kinneberg for patient recruitment; Ms. Cathy Bagne for coordinator support; and Ms. Patricia L. Erickson and Ms. Tanya Doble for helping with manuscript preparation. We greatly appreciate all the subjects who volunteered for these renal biopsy studies.

\section{Disclosure Statement}

This work was supported by the National Institutes of Health (NIH) grant P01-DK13083-08 and funds from the Pennock Professorship and the Minnesota Lions Diabetes Foundation (M.L.C., principal investigator). M.L.C. was a recipient of a Career Development Award from the Juvenile Diabetes Research Foundation (JDRF).

The results presented in this paper have not been published previously in whole or part, except in an abstract format.

\section{References}

1 Caramori ML, Fioretto P, Mauer M: The need for early predictors of diabetic nephropathy risk: is albumin excretion rate sufficient? Diabetes 2000;49:1399-1408.

2 Caramori ML, Kim Y, Huang C, Fish AJ, Rich SS, Miller ME, Russell G, Mauer M: Cellular basis of diabetic nephropathy: 1 . Study design and renal structural-functional relationships in patients with long-standing type 1 diabetes. Diabetes 2002;51:506-513.

3 Fioretto P, Steffes MW, Mauer M: Glomerular structure in nonproteinuric IDDM patients with various levels of albuminuria. Diabetes 1994;43:1358-1364.
4 Lane PH, Steffes MW, Mauer SM: Glomerular structure in IDDM women with low glomerular filtration rate and normal urinary albumin excretion. Diabetes 1992;41:581-586.

5 Caramori ML, Fioretto P, Mauer M: Low glomerular filtration rate in normoalbuminuric type 1 diabetic patients: an indicator of more advanced glomerular lesions. Diabetes 2003; 52:1036-1040.

6 Torbjörnsdotter TB, Perrin NE, Jaremko GA, Berg UB: Widening of foot processes in normoalbuminuric adolescents with type 1 diabetes. Pediatr Nephrol 2005;20: 750-758.
7 Perrin NE, Torbjörnsdotter TB, Jaremko GA, Berg UB: Follow-up of kidney biopsies in normoalbuminuric patients with type 1 diabetes. Pediatr Nephrol 2004;19:10041013.

8 Bjørn SF, Bangstad HJ, Hanssen KF, Nyberg G, Walker JD, Viberti GC, Osterby R: Glomerular epithelial foot processes and filtration slits in IDDM patients. Diabetologia 1995;38:1197-1204.

9 Ellis EN, Steffes MW, Chavers B, Mauer SM: Observations of glomerular epithelial cell structure in patients with type I diabetes mellitus. Kidney Int 1987;32:736-741. 
10 Toyoda M, Najafian B, Kim Y, Caramori ML, Mauer M: Podocyte detachment and reduced glomerular capillary endothelial fenestration in human type 1 diabetic nephropathy. Diabetes 2007;56:2155-2160.

11 Pagtalunan ME, Miller PL, Jumping-Eagle S, Nelson RG, Myers BD, Rennke HG, Coplon NS, Sun L, Meyer TW: Podocyte loss and progressive glomerular injury in type II diabetes. J Clin Invest 1997;99:342-348.

12 White KE, Bilous RW: Structural alterations to the podocyte are related to proteinuria in type 2 diabetic patients. Nephrol Dial Transplant 2004;19:1437-1440.

13 Dalla Vestra M, Masiero A, Roiter AM, Saller A, Crepaldi G, Fioretto P: Is podocyte injury relevant in diabetic nephropathy? Studies in patients with type 2 diabetes. Diabetes 2003; 52:1031-1035.

14 Meyer TW, Bennett PH, Nelson RG: Podocyte number predicts long-term urinary albumin excretion in Pima Indians with type II diabetes and microalbuminuria. Diabetologia 1999;42:1341-1344.

15 Lemley KV, Abdullah I, Myers BD, Meyer TW, Blouch K, Smith WE, Bennett PH, Nelson RG: Evolution of incipient nephropathy in type 2 diabetes mellitus. Kidney Int 2000; 58:1228-1237.

16 Steffes MW, Schmidt D, McCrery R, Basgen JM: Glomerular cell number in normal subjects and in type 1 diabetic patients. Kidney Int 2001;59:2104-2113.

17 White KE, Bilous RW, Marshall SM, El Nahas M, Remuzzi G, Piras G, De Cosmo S, Viberti G: Podocyte number in normotensive type 1 diabetic patients with albuminuria. Diabetes 2002;51:3083-3089.
18 Caramori ML, Parks A, Mauer M: Renal lesions predict progression of diabetic nephropathy in type 1 diabetes. J Am Soc Nephrol 2013;24:1175-1181.

19 Chavers BM, Simonson J, Michael AF: A solid phase fluorescent immunoassay for the measurement of human urinary albumin. Kidney Int 1984;25:576-578.

20 Gaspari F, Perico N, Matalone M, Signorini O, Azzollini N, Mister M, Remuzzi G: Precision of plasma clearance of iohexol for estimation of GFR in patients with renal disease. J Am Soc Nephrol 1998;9:310-313.

21 Mauer M, Drummond K: The early natural history of nephropathy in type 1 diabetes: I. Study design and baseline characteristics of the study participants. Diabetes 2002;51: 1572-1579.

22 Ellis EN, Steffes MW, Goetz FC, Sutherland DE, Mauer SM: Glomerular filtration surface in type I diabetes mellitus. Kidney Int 1986; 29:889-894.

23 Camargo JL, Zelmanovitz T, Paggi A, Friedman R, Gross JL: Accuracy of conversion formulae for estimation of glycohaemoglobin. Scand J Clin Lab Invest 1998;58:521528.

24 Jensen EB, Gundersen HJ, Osterby R: Determination of membrane thickness distribution from orthogonal intercepts. J Microsc 1979; 115:19-33.

25 Steffes MW, Bilous RW, Sutherland DE, Mauer SM: Cell and matrix components of the glomerular mesangium in type I diabetes. Diabetes 1992;41:679-684.
26 Mauer SM, Steffes MW, Ellis EN, Sutherland DE, Brown DM, Goetz FC: Structural-functional relationships in diabetic nephropathy. J Clin Invest 1984;74:1143-1155.

27 Berg UB, Torbjörnsdotter TB, Jaremko G, Thalme B: Kidney morphological changes in relation to long-term renal function and metabolic control in adolescents with IDDM. Diabetologia 1998;41:1047-1056.

28 Osterby R: Glomerular structural changes in type 1 (insulin-dependent) diabetes mellitus: causes, consequences, and prevention. Diabetologia 1992;35:803-812.

29 Chavers BM, Bilous RW, Ellis EN, Steffes MW, Mauer SM: Glomerular lesions and urinary albumin excretion in type I diabetes without overt proteinuria. N Engl J Med 1989; 320:966-970

30 Drummond KN, Kramer MS, Suissa S, LévyMarchal C, Dell'Aniello S, Sinaiko A, Mauer M: Effects of duration and age at onset of type 1 diabetes on preclinical manifestations of nephropathy. Diabetes 2003;52:1818-1824.

31 Ellis EN, Warady BA, Wood EG, Hassanein R, Richardson WP, Lane PH, Howard C, Kemp SF, Aceto T, Garibaldi L, Wiegmann TB, Savin VJ: Renal structural-functional relationships in early diabetes mellitus. Pediatr Nephrol 1997;11:584-591.

32 Perrin NE, Torbjörnsdotter T, Jaremko GA, Berg UB: Risk markers of future microalbuminuria and hypertension based on clinical and morphological parameters in young type 1 diabetes patients. Pediatr Diabetes 2010;11: 305-313.

33 Miller PL, Meyer TW: Effects of tissue preparation on glomerular volume and capillary structure in the rat. Lab Invest 1990;63:862-866. 